\title{
Canadian Revue
}

Journal of canadienne de
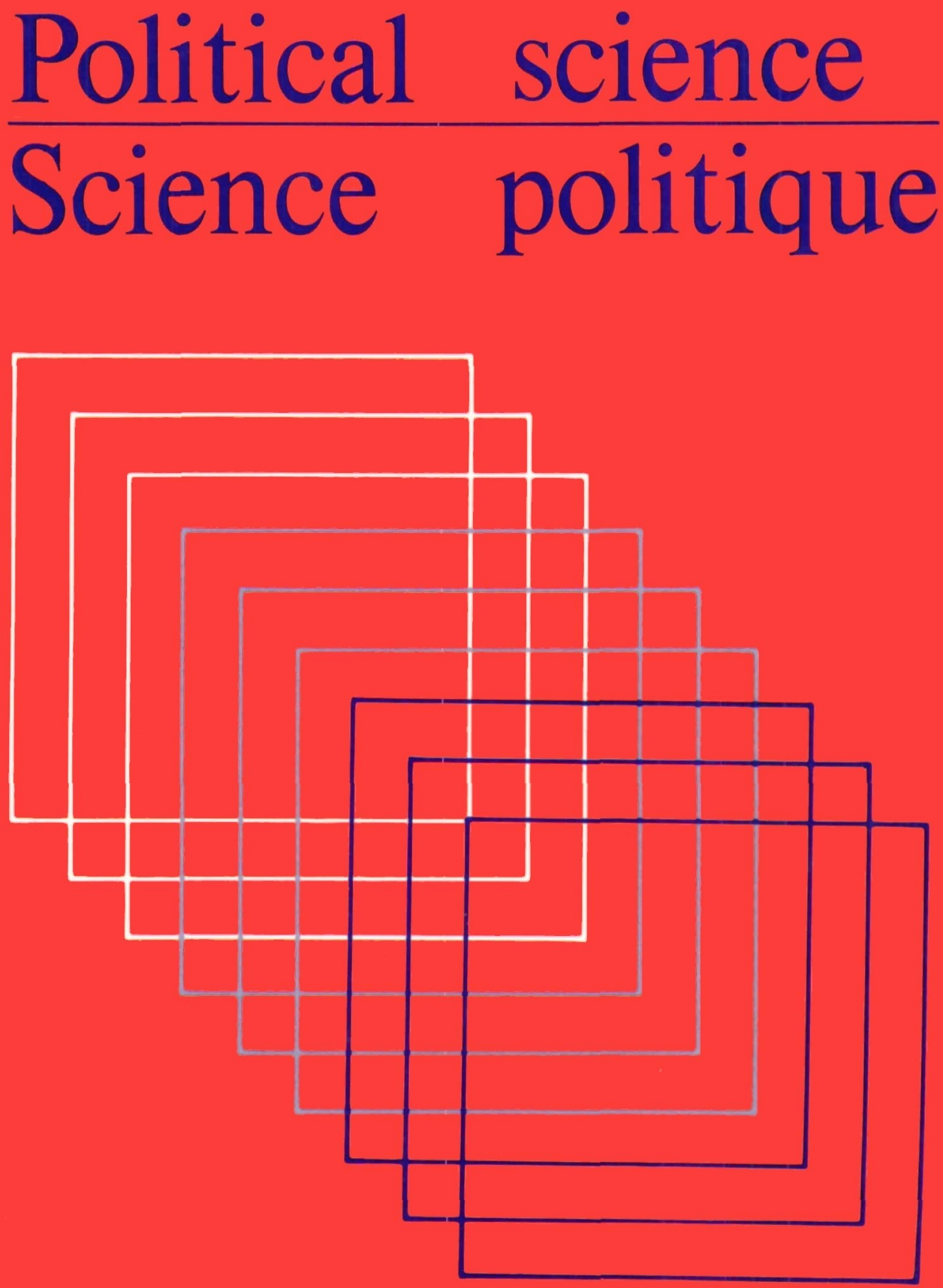

XXVII:4 December 1994 décembre 


\section{Canadian Journal of Political Science / Revue canadienne de science politique}

The Journal acknowledges with gratitude the generous assistance of the Social Sciences and Humanities Research Council of Canada. / La Revue tient à remercier le Conseil de recherches en sciences humaines du Canada pour la généreuse subvention qu'il a bien voulu lui accorder.

The Journal also acknowledges support from / La Revue bénéficie aussi du soutien de: Carleton University, l'Université Laval, University of Manitoba, University of Western Ontario, Wilfrid Laurier University.

Membership in the Canadian Political Science Association and/or the Société québécoise de science politique includes a subscription to the Journal. Institutional subscriptions (which do not include membership privileges) are also available. The Journal may be obtained in paper and fiche. / La cotisation versée à l'Association canadienne de science politique et/ou a La société québécoise de science politique donne droit à un abonnement à La Revue. Des abonnements déstinés aux institutions (qui ne donnent pas droit aux privilèges réservés aux membres) sont également offerts. Vous pouvez recevoir La Revue sous la forme d'une revue et de micro-fiches.

\begin{tabular}{ll}
$\begin{array}{l}\text { Students and any other person } \\
\text { earning under } \$ 30,000 \text { annually/ } / \\
\text { Étudiants et tout autre } \\
\text { personne dont le revenu annuel } \\
\text { ne dépasse pas } 30000 \$\end{array}$ & $\begin{array}{l}\text { All other } \\
\text { members/ } \\
\text { Tous les } \\
\text { autres } \\
\text { membres }\end{array}$ \\
\hline$\$ 35.00$ & $\$ 100.00$ \\
$\$ 35.00$ & $\$ 95.00$ \\
$\$ 45.00$ & $\$ 110.00$
\end{tabular}

The choice of Associations/

Choix d'associations

$\$ 110.00$

\begin{tabular}{lcc}
\hline CPSA only / ACSP seulement & $\$ 35.00$ & $\$ 100.00$ \\
SQSP seulement / only* & $\$ 35.00$ & $\$ 95.00$ \\
Joint/conjoint CPSA/ACSP-SQSP* & $\$ 45.00$ & $\$ 110.00$ \\
* These categories of membership include a subscription to the SQSP's journal \\
Revue québécoise de science politique. / Ces catégories d'adhésion compren- \\
nent un abonnement à la Revue québécoise de science politique de la SQSP. \\
\hline Institutional subscription - $\$ 65.00$ in Canada; $\$ 85.00$ in member countries of OECD; \\
\$65.00 in all other countries \\
Abonnement institutionnel - $65 \$$ au Canada; $85 \$$ dans les pays-membres de l'OCDE; \\
$65 \$$ dans tout autre pays
\end{tabular}

Business correspondence should be sent to: Executive Secretary, Canadian Political Science Association, Suite 205, 1 Stewart Street, Ottawa, Ontario KIN 6H7 (e-mail: CPSCC@ACADVM1. UOTTAWA.CA; tel.: 1-613-564-4026; fax: 1-613-230-2746)./ Prière d' adresser toute communication de caractère commercial à: Secrétaire administrative, Association canadienne de science politique, Bureau 205, 1, rue Stewart, Ottawa, Ontario K1N 6H7 (adresse électronique : CPSCC@ ACADVM1.UOTTAWA.CA; téléphone : 1-613-564-4026; télécopieur : 1-613-230-2746).

The headquarters of the Canadian Political Science Association is Suite 205, 1 Stewart Street, Ottawa. Ontario K1N 6H7. / Le siège social de l'Association canadienne de science politique est Bureau 205, 1, rue Stewart, Ottawa, Ontario KIN 6H7. Le siège social de la Société québécoise de science politique est à / The headquarters of the Société québécoise de science politique is at the Université du Québec à Montréal, Montréal, Québec H3C 3P8.

Published quarterly by / Publiée quatre fois par an à Wilfrid Laurier University Press for/pour the Canadian Political Science Association (l'Association canadienne de science politique) and/et la Société québécoise de science politique.

- Canadian Political Science Association (l'Association canadienne de science politique) and/et la Société québécoise de science politique December/décembre 1994 (date of issue/date de parution : February/février 1995)

CN ISSN 0008-4239

Publications Mail Registration / Poste-publications enregistrement \#4011

Return postage guaranteed / Port de retour garanti

$\infty$ 
XXVII:4 December 1994 décembre

Facing a Renewed Right: American Feminism and the

Reagan/Bush Challenge

SYLVIA BASHEVKIN 669

Télévision et unité nationale : la couverture de l'actualité canadienne à Radio-Canada, 1977 et 1987

JEAN DE BONVILLE et JACQUES VERMETTE 699

What Kind of Democracy Do Canadians Want?

MICHAEL M. ATKINSON 717

Constitutional Paralysis: Why Canadian Constitutional Initiatives Are Doomed to Fail

MICHAEL LUSZTIG 747

How Do Peaceful Secessions Happen?

ROBERT A. YOUNG 773

Recensions/Reviews 793

Notices / Avis 881

Index 885 
Editorial Board / Comité de rédaction

Richard Vernon (Western Ontario), co-editor

Kathy L. Brock (Manitoba), review editor

Sid Noel (Western Ontario), assistant editor
Guy Laforest (Laval), codirecteur

François Rocher (Carleton), secrétaire à la rédaction et responsable des recensions

Administrative Editor / Directeur administratif John McMenemy (Wilfrid Laurier)

Advisory Board / Conseil consultatif

Gerald Bernier (Montréal)

Alan C. Cairns (British Columbia)

Réjean Landry (Laval)

Alexander MacLeod (UQAM)
Mildred Schwartz(Illinois)

Jennifer Smith (Dalhousie)

Reg Whitaker (York)

The Canadian Journal of Political Science, whose policy is set by the Editorial Board, is committed to the publication of scholarly research in all fields of political science. Manuscripts are assessed on the basis of their contribution to the discipline and by the authors' adherence to the highest scholarly standards.

La Revue canadienne de science politique dont la politique est mise en place par le comité de rédaction s'engage à publier des travaux de recherche académique dans toutes les orientations de la science politique. Les manuscrits sont évalués sur la base de leur contribution à l'avancement des connaissances dans la discipline et selon la conformité des textes aux exigences académiques les plus élévées.

See Notes to Contributors on the inside back cover. / Voir avis aux collaborateurs sur la troisième de couverture.

INDEXED IN / INDEXÉE AU: International Political Science Abstracts/Documentation politique internationale; $A B C$ POL SCI (Advance Bibliography of Contents: Political Science and Government); Historical Abstracts; America: History and Life; Political Science Abstracts; Point de Repère: index analytique d'articles de périodiques de langue française; Academic Abstracts; Social Science Source; Social Sciences Index; Sociological Abstracts (partial/partiel); United States Political Science Documents; Arts \& Humanities Citation Index (A\&HCI); Current Contents/Social and Behavioral Sciences; Social Sciences Citation Index; Social Scisearch; Research Alert; PAIS Bulletin and PAIS Foreign Language Index; Canadian Periodical Index/Index de périodiques canadiens; Canadian Magazine Index and available on-line in the Canadian Business and Current Affairs Database; Index to Canadian Legal Literature/Index à la documentation juridique au Canada (partial/partiel); Index to Canadian Legal Periodical Literature (partial/partiel); Indian Book Review Digest (partial/partiel); International Political Science Information Service; IBZ (Internationale Bibliographie der Zeitschriftenliteratur);IBR (Internationale Bibliographie der Rezensionen). 